\title{
Epigenetic and developmental influences on the risk of obesity, diabetes, and metabolic syndrome
}

This article was published in the following Dove Press journal:

Diabetes, Metabolic Syndrome and Obesity: Targets and Therapy

29 June 2015

Number of times this article has been viewed

\author{
Caitlin J Smith \\ Kelli K Ryckman \\ Department of Epidemiology, \\ University of lowa, College of Public \\ Health, lowa City, IA, USA
}

Correspondence: Kelli K Ryckman

Department of Epidemiology, University of lowa, College of Public Health, 145 Riverside Drive, S4I4 CPHB, lowa City, IA 52242, USA

$\mathrm{Tel}+\mathrm{I} 3193841562$

Fax +I 3193844155

Email kelli-ryckman@uiowa.edu

\begin{abstract}
Metabolic syndrome is a growing cause of morbidity and mortality worldwide. Metabolic syndrome is characterized by the presence of a variety of metabolic disturbances including obesity, hyperlipidemia, hypertension, and elevated fasting blood sugar. Although the risk for metabolic syndrome has largely been attributed to adult lifestyle factors such as poor nutrition, lack of exercise, and smoking, there is now strong evidence suggesting that predisposition to the development of metabolic syndrome begins in utero. First posited by Hales and Barker in 1992, the "thrifty phenotype" hypothesis proposes that susceptibility to adult chronic diseases can occur in response to exposures in the prenatal and perinatal periods. This hypothesis has been continually supported by epidemiologic studies and studies involving animal models. In this review, we describe the structural, metabolic and epigenetic changes that occur in response to adverse intrauterine environments including prenatal and postnatal diet, maternal obesity, and pregnancy complications. Given the increasing prevalence of metabolic syndrome in both the developed and developing worlds, a greater understanding and appreciation for the role of the intrauterine environment in adult chronic disease etiology is imperative.
\end{abstract}

Keywords: epigenetics, metabolic syndrome, fetal programming, maternal, pregnancy complications

\section{Introduction}

Metabolic syndrome encompasses a complex network of symptoms and risk factors. It is defined by the National Institutes of Health (NIH) by having at least three of the following conditions: central obesity, elevated triglycerides, low high-density lipoprotein cholesterol, hypertension or elevated fasting plasma glucose. Although it is widely accepted that lifestyle factors such as obesity, high caloric diet, and smoking contribute to the development of metabolic syndrome by virtue of their role in diabetes and cardiovascular disease, substantial research supports the role of early life exposures in the etiology of metabolic syndrome and related disorders including obesity and type 2 diabetes (T2D).

Historical epidemiologic observations have led to further study of the association between the intrauterine environment and subsequent disease. Most notably, the Dutch famine of 1944-1945 led to the epidemiologic observation that children born to mothers who were in the early stages of pregnancy during the famine were at significantly increased risk of cardiometabolic disorders in adulthood. ${ }^{1}$ Hales and Barker observed the relationship between low birth weight and increased risk for T2D in a British cohort, leading to the proposal of the "thrifty phenotype" hypothesis, which posited that malnutrition during pregnancy results in structural and functional changes in the developing fetus. ${ }^{2}$ 
Since the proposal of the thrifty phenotype hypothesis in 1992, numerous studies have replicated the finding that low birth weight and other pregnancy complications confer increased risk for adult chronic disease in the offspring, including hypertension, T2D, and cardiovascular disease. ${ }^{3-6}$ This concept has evolved into the "developmental origins of adult disease" hypothesis, to encompass the interplay between an individual's genetic background and the intrauterine environment that results in permanent metabolic changes that impact the risk for later life chronic disease. ${ }^{7}$ Changes occurring during critical periods of development, such as embryonic and fetal development, that promote survival of the fetus in an adverse intrauterine environment, such as maternal malnutrition, can also have lasting effects on the individual's metabolic health, increasing their risk of chronic disease later in life. These "programmed" changes or alterations, referred to here as developmental programming, can result in permanent structural changes of organs and tissues, altered responses to environmental stimuli, and epigenetic changes in gene expression.

While the exact molecular mechanisms underlying developmental programming are largely unknown, there are likely multiple interrelated interactions between genetics and the intrauterine environment that when occurring during critical periods of fetal development lead to permanent metabolic and vascular changes impacting individual risk for later life chronic disease. At the structural level, intrauterine exposures may damage developing cells or tissues. At the chemical level, exposures may manipulate cellular responses to stimuli. When combined, these developmental insults can result in increased risk for metabolic syndrome and other chronic diseases including obesity and T2D. ${ }^{8,9}$

Although developmental programming is typically thought to refer only to responses to the intrauterine environment, it may also occur prior to conception by the programming of gametes. In particular, maternal metabolic syndrome can affect the quality of oocytes and embryos, ${ }^{10,11}$ which can program the resulting offspring to have an increased risk of developing metabolic syndrome.

Animal models of intrauterine exposures and pregnancy complications and the resulting changes in the offspring, including structural and epigenetic, have been particularly valuable for studying the developmental origins of chronic diseases from a developmental programming perspective. ${ }^{12}$ In particular, rodent models of maternal obesity and insulin resistance, nutrition intervention, intrauterine growth restriction (IUGR), and early postnatal growth provide a rich source of evidence for the developmental programming of metabolic syndrome and other metabolic disorders.
In this review, we describe human and animal studies that support the role of developmental programming in the etiology of metabolic syndrome, obesity, and T2D. Specifically, we focus on how the developmental programming of metabolic disorders are influenced by epigenetic programming, maternal and postnatal diet, maternal obesity, and pregnancy complications.

\section{Epigenetic programming}

The metabolic disturbances triggered by adverse intrauterine conditions are often accompanied by epigenetic changes. Epigenetics refers to the changes in the biochemical structure of DNA that alter gene expression. These changes include, but are not limited to, DNA methylation, histone modification, and non-coding RNA processes..$^{13}$ The epigenetic state of an individual's DNA is both inherited and modifiable, such that DNA expression patterns can be passed from parent to offspring or they can be modified in response to environmental triggers. ${ }^{14}$ Epigenetic changes have been observed in association with many disease states, and many are observed in metabolic syndrome and its encompassing conditions. ${ }^{15}$ Thus, epigenetic changes offer biochemical evidence of the deleterious effects of adverse developmental conditions and subsequent disease.

Methylation of $5^{\prime}$ cytosine residues at cytosine/guaninerich regions of DNA (also known as $\mathrm{CpG}$ islands) is a common epigenetic modification and hypermethylation of these regions is associated with transcriptional repression. ${ }^{16}$ This repression may be mediated by histone modifications, in which hypermethylated $\mathrm{CpG}$ islands promote the binding of MECP2, which recruit histone modifying complexes. ${ }^{16}$ The ensuing histone modification results in a chromatin structure that is inaccessible to transcription machinery.

Throughout this review, we describe epigenetic changes occurring as a consequence of maternal and postnatal diet, maternal obesity, and pregnancy complications. Furthermore, we discuss how these epigenetic changes are involved in the offspring's risk for developing metabolic syndrome in adulthood.

\section{Maternal and early postnatal diet}

The relationship between the intrauterine environment and chronic disease in the offspring was first noted in response to maternal malnutrition. It is hypothesized that in the presence of maternal malnutrition the developing fetus' metabolism is structurally and functionally altered in anticipation of harsh conditions after birth. This adaptation can have even greater detrimental effects on the offspring when the postnatal environment is nutrient-rich, rather than nutrient poor. ${ }^{17}$ 
Manipulation of the maternal diet in mice and rats has illustrated the effects of in utero nutrition on the metabolic health of the offspring. Ponzio et al has described the transgenerational effects of maternal nutrient restriction on hypertension and endothelial dysfunction in rats. ${ }^{18}$ Female pregnant rats (F0) were exposed to $50 \%$ nutrient deprivation and cardiovascular factors were measured in their offspring (F1) and subsequent generations (F2 and F3) that were fed normal non-restrictive diets. High blood pressure and other metabolic perturbations were observed in all offspring born to nutrient restricted mothers compared to offspring of mothers who were not nutrient restricted. These results demonstrate the transmission of cardiovascular perturbations across multiple generations in response to a single incidence of maternal nutrient restriction. ${ }^{18}$

Maternal over-nutrition has been shown to have equally detrimental effects on the future health of the offspring. ${ }^{19,20}$ As with maternal malnutrition, over-nutrition programs the developing fetus to anticipate excessive nutrient availability after birth. Excess caloric intake during pregnancy is arguably more relevant to today's society, in which "junk food" is readily available in most developed and developing regions of the world. ${ }^{21}$ Thus, it is critical to achieve greater understanding of the mechanisms of these effects as they may offer insights into the rising incidence of metabolic diseases, including obesity, T2D, and metabolic syndrome.

Many animal studies have investigated the effects of maternal over-nutrition on the metabolic health of the offspring. Both high-fat and high-calorie diets have been shown to increase adiposity and inflammation in the offspring. ${ }^{22}$ Endothelial dysfunction has been observed in response to maternal high-fat diet in nonhuman primates. ${ }^{23}$ Female Japanese macaques were exposed to either a control diet or high-fat diet for up to 5 years and their offspring were exposed to either a control or high-fat diet post-weaning. Metabolic and cardiovascular markers were characterized in offspring at 13 months of age. Animals born to high-fat diet-fed mothers and who were fed a high-fat diet post-weaning $(\mathrm{H} / \mathrm{H})$ displayed the most perturbation. They demonstrated elevated fasting insulin levels, although fasting glucose was normal, indicating the early stages of insulin resistance. $\mathrm{H} / \mathrm{H}$ animals also demonstrated impaired acetylcholine-induced vasodilation, an indicator of endothelial dysfunction. Animals born to high-fat diet-fed mothers who were exposed to normal diet post-weaning demonstrated slight metabolic and endothelial perturbations compared to offspring born to control-fed mothers and fed a normal diet after birth. ${ }^{23}$ The results of this study indicate that the effects of maternal high-fat diet are most detrimental in the presence of a postnatal high-fat diet; however, a healthy or normal postnatal diet cannot completely reverse the metabolic effects caused by poor maternal nutrition. ${ }^{23}$ This is further supported by a study in rats in which females were fed a high-fat diet during pregnancy and offspring were fed a normal control diet post-weaning; the offspring maintained an adverse metabolic profile and epigenetic changes through adulthood, suggesting that irreversible metabolic damage in the offspring can be caused by poor maternal nutrition during pregnancy. ${ }^{24}$

The biological mechanisms underlying the association between maternal nutrition and later life metabolic health in the offspring are unclear. Leptin has been implicated as a possible mediator of increased risk for obesity in response to adverse intrauterine conditions..$^{25}$ Leptin is a hormone secreted by adipose tissue and is active during both fetal and postnatal life. In utero, leptin is supplied via maternal blood and acts as a neurotrophic factor to aid in development of the central nervous system. ${ }^{25}$ In postnatal life, leptin regulates appetite and energy expenditure to maintain energy balance. ${ }^{26}$ Evidence from animal studies demonstrates that leptin sensitivity in offspring is affected by maternal diet, ${ }^{27}$ and these changes can have transgenerational effects..$^{27,28}$ Studies in rodents demonstrate the adverse effects of both maternal malnutrition and high-fat diet on leptin sensitivity. In the case of maternal malnutrition, the reduction in circulating maternal leptin results in decreased leptin sensitivity and obesity in the offspring. ${ }^{29}$ Rat offspring exposed to a high-fat maternal diet demonstrated leptin resistance in adulthood regardless of post-weaning diet. ${ }^{30}$ In another study of rats exposed to maternal hyperglycemia, male offspring who were fed a high-fat diet demonstrated significantly increased leptin levels compared to male offspring not exposed to maternal hyperglycemia. ${ }^{31}$ Female offspring exposed to maternal hyperglycemia and fed a high-fat diet demonstrated only moderate increase in leptin levels compared to female offspring not exposed to maternal hyperglycemia. ${ }^{31}$ This evidence supports the programming of leptin sensitivity in response to maternal diet, with potentially sex-specific effects. Furthermore, this research suggests leptin as a mediator of the association between intrauterine exposures and metabolic disturbances in the offspring.

In addition to the role of leptin, adiponectin (ADIPOQ) has been implicated in metabolic syndrome. ${ }^{32}$ In mice exposed to maternal high-fat diet, serum ADIPOQ-1 and ADIPOQ receptor expression in skeletal muscle expression were significantly lower than controls. Offspring of mothers fed high-fat diets grew to be significantly heavier and had 
significantly elevated fasting glucose levels compared to mice exposed to a normal maternal diet. ${ }^{33}$ Given that ADIPOQ helps to maintain insulin sensitivity and ADIPOQ levels are inversely correlated with obesity, the results of this study suggest that metabolic disturbances in response to maternal high-fat diet may also be mediated by ADIPOQ. ${ }^{33}$

\section{Maternal obesity, insulin resistance, and metabolic syndrome}

Maternal health prior to and at the time of conception can have detrimental effects on the pregnancy and the subsequent health of the child. In particular, obesity and insulin resistance during pregnancy have been consistently shown to negatively impact the metabolic health of the offspring. Obesity and insulin resistance often co-exist and are common metabolic conditions of pregnancy with an estimated $33 \%$ of all pregnancies complicated by maternal obesity. The relationship between obesity and insulin resistance in pregnancy and the impact on obesity, T2D, and metabolic syndrome in the offspring may be due to permanent alterations in glucose-insulin metabolism in the offspring, causing reduced capacity for insulin secretion and insulin resistance. ${ }^{23}$ In fact, maternal obesity and insulin resistance have been shown to confer insulin resistance as early as the embryonic stage in animal models. ${ }^{10}$ Maternal insulin resistance can negatively impact the developing embryo due to impaired glucose transport. ${ }^{34}$

In a study of the umbilical cords of lean pregnant women compared to obese pregnant women, maternal obesity was associated with significantly increased leptin and insulin levels in maternal plasma and cord plasma. ${ }^{35}$ These differences were accompanied by differential umbilical cord gene expression between lean and obese mothers. A study of 99 offspring of diabetic mothers evaluated cardiovascular risk factors in childhood and adolescence. They observed a significant increase in E-selectin, vascular cell adhesion molecule 1 (VCAM1), and leptin levels in offspring of diabetic mothers compared to offspring of non-diabetic mothers, increased body mass index and waist circumference and decreased ADIPOQ. E-selectin and VCAM1 are markers of endothelial function and atherosclerosis and leptin and ADIPOQ are regulators of hunger and metabolism. ${ }^{36}$ Increased leptin and decreased ADIPOQ levels are likely due to the increased body mass index and waist circumference. ${ }^{36}$

Gestational diabetes mellitus (GDM) is characterized by novel hyperglycemia during pregnancy. ${ }^{37}$ In addition to increasing the maternal risk of T2D and metabolic syndrome, GDM is consistently shown to increase the risk of obesity,
$\mathrm{T} 2 \mathrm{D}$, and metabolic syndrome in the offspring both in adolescence and adulthood. ${ }^{38}$ The increased risk for metabolic disturbances is thought to be mediated by oxidative stress. Elevated maternal blood glucose triggers the production of reactive oxygen species. These substances are normally neutralized by antioxidants, but the antioxidant profiles of mothers with GDM and their offspring are altered. ${ }^{39}$ Similarly, GDM is associated with hyperlipidemia in both mother and neonate, which is consistent with the observation of hyperlipidemia in T2D and metabolic syndrome. ${ }^{40}$

Maternal obesity and diabetes are associated with epigenetic changes in the offspring, which mediate the metabolic disturbances seen in these offspring. In a mouse model of obesity and T2D, triglyceride and leptin levels were significantly elevated in offspring of obese/T2D mothers compared to offspring of mothers with normal metabolism. ${ }^{41}$ These changes were accompanied by reduced expression of two mitochondrial genes, ATPASE6 and CYTB, and widespread changes in methylation patterns. These changes were exacerbated in offspring of obese mothers who were then fed a high-fat diet postnatally, compared to offspring fed a normal diet. The results of this study provide further evidence of the effect of the maternal metabolic state on the metabolic health of the offspring, and demonstrate the additionally detrimental effects of a high-fat postnatal diet following exposure to maternal obesity. ${ }^{41}$

GDM has been shown to trigger epigenetic modifications, including alterations in telomere length. ${ }^{42}$ In a study of offspring of women with GDM, telomere length was significantly shorter in fetuses of women with GDM compared to fetuses of women with uncomplicated pregnancy. ${ }^{42}$ In adulthood, shortened telomere length is associated with increased risk of cardiometabolic diseases. Thus, the finding that telomere length is significantly shorter in developing fetuses exposed to GDM suggests a genetic mechanism for the increased risk of cardiometabolic disorders seen in these children that begins in utero.

Animal evidence of developmental programming suggests that sex differences exist in the susceptibility to the detrimental effects of intrauterine exposures. ${ }^{43,44}$ In mice exposed to pre-conceptional and gestational obesity, male but not female offspring developed obesity, insulin resistance, hyperleptinemia, and hepatic steatosis. ${ }^{43}$ Differential expression of the lipogenic genes, Srebf1, Fasn, Pparg1 and Pparg2 was observed between male and female offspring, in which males exposed to pre-conceptional and gestational obesity demonstrated significantly different expression compared to control males, whereas no significant difference was 
observed between exposed females compared to control females. ${ }^{43}$ Conversely, females showed significant increases in adipocyte size compared to control females, with no significant differences among males. These sex differences in adipocyte size were accompanied by differential expression of genes regulating lipid droplet size and lipogenesis, Pnpla 2 and $\mathrm{Bscl} 2$ and a gene involved in apoptosis, Bax, among exposed females compared to control females, but not in males. ${ }^{43}$ The results of this study demonstrate sex differences in the metabolic and epigenetic response to pre-conceptional and gestational obesity. ${ }^{43}$

In a study of the effects of maternal obesity, as triggered by a maternal high-fat diet, mice of both sexes that were exposed to maternal obesity developed hypercholesterolemia, hyperinsulinemia, and hyperleptinemia. ${ }^{44}$ However, exposed male offspring, as compared to exposed female offspring, had more severe hypercholesterolemia and hyperinsulinemia but a similar degree of hyperleptinemia. The results of this study provide further evidence of the detrimental effects of maternal obesity on cholesterol and insulin sensitivity in the offspring, which are manifested in a sex-specific manner. ${ }^{44}$

In a study of male mice exposed to maternal obesity, glucose tolerance was not impaired in maternal obesity offspring compared to control offspring, although fasting insulin levels were significantly elevated in obese offspring. ${ }^{45}$ Interestingly, expression levels of insulin receptor substrate (IRS-1) was markedly reduced in maternal obesity offspring, while levels of $m i R-126$, a micro-RNA known to inhibit translation of IRS-1, were markedly increased. This suggests that hyperinsulinemia in response to maternal obesity is mediated by IRS-1, which is under epigenetic regulation through a micro-RNA, $m i R-126 .{ }^{45}$ Taken together, the aforementioned evidence demonstrates the influence of maternal metabolic health on the metabolic health of the offspring.

\section{Pregnancy complications, low birth weight, and prematurity}

Pregnancy complications and resulting perinatal outcomes are widely accepted to increase the risk of adult chronic disease in the offspring, including risk for cardiovascular disease, T2D, and metabolic syndrome. ${ }^{46}$ Such complications include gestational hypertension, preeclampsia, and preterm birth.

Gestational hypertension and preeclampsia have been associated with development of chronic disease in the offspring including hypertension and cardiovascular disease. ${ }^{8,47,48}$ Gestational hypertension is characterized by novel onset of hypertension during pregnancy while preeclampsia is characterized by novel onset of hypertension during pregnancy, in addition to proteinuria. ${ }^{48}$ The 1986 Northern Finland Birth Cohort study investigated the effect of gestational hypertension on the blood pressure of the offspring in adolescence. ${ }^{48}$ Children of mothers with gestational hypertension had significantly elevated blood pressure compared to children of normotensive mothers. Another study, using data from the Avon Longitudinal Study of Parents and Children (ALSPAC), investigated the effects of maternal gestational hypertension and preeclampsia on the blood pressure of the offspring in childhood. ${ }^{49}$ They found that gestational hypertension was associated with significantly increased blood pressure in the children, aged 9-12, while preeclampsia showed no association when adjusted for other factors. A study of women with preeclamptic pregnancies and their children demonstrated the deleterious effects of preeclampsia on the cardiovascular health of the child. Women and children were screened for cardiovascular biomarkers 5-8 years postpartum compared to women and children of uncomplicated pregnancies. ${ }^{50}$ They observed significantly elevated total cholesterol in children exposed to preeclampsia compared to children exposed to uncomplicated pregnancies.

Preeclampsia has also been shown to induce epigenetic modifications in the offspring. In a study of the effects of preeclampsia and its effects on DNA methylation, umbilical cord methylation at $I G F 2$ was significantly lower among infants exposed to preeclampsia compared to infants exposed to uncomplicated pregnancy. Methylation of IGF2 was inversely correlated with birth weight. ${ }^{51}$ Given that $I G F 2$ is a regulator of fetal growth, this study suggests that low birth weight as a result of preeclampsia may be mediated by $I G F 2$.

Pregnancy complications can result in offspring born large for gestational age, often referred to as macrosomia, or offspring born small for gestational age. Small for gestational age is often a result of IUGR. IUGR manifests as low birth weight and is generally characterized by birth weight below the 10th percentile, ${ }^{12}$ and has repeatedly been shown to negatively impact health in adulthood. IUGR can be the result of many conditions, including placental pathologies, intrauterine infections, preeclampsia or gestational hypertension, fetal syndromes, smoking, alcohol or substance abuse, severe malnutrition, maternal autoimmune diseases or severe maternal T2D. ${ }^{12}$ Conversely, macrosomia, characterized by birth weight above the 90th percentile, can be equally as detrimental to future health. Potential known causes of macrosomia include poorly managed maternal T2D, prolonged pregnancy, 
history of macrosomic pregnancy, or polyhydramnios. ${ }^{12}$ Both IUGR and macrosomia are associated with increased risk of metabolic syndrome. ${ }^{52,53}$ Rat models of IUGR, caused by a decrease in placental blood flow, have demonstrated metabolic and epigenetic changes in the offspring. ${ }^{54,55} \mathrm{~A}$ study demonstrated that IUGR caused insulin resistance in a sex-specific manner, in which growth-restricted females had significantly higher fasting blood glucose levels than control females, whereas growth-restricted males showed no significant difference. ${ }^{56}$

Growth restriction can also be the result of maternal malnutrition, and the postnatal environment following IUGR can impact the metabolic profile in adulthood. ${ }^{57}$ Sheep exposed to maternal caloric restriction to induce IUGR followed by exposure to a low-activity, high calorie postnatal environment to induce rapid catch-up growth demonstrated obesity, insulin and leptin resistance, and elevated cortisol levels. In contrast, IUGR offspring exposed to a normal postnatal environment to induce normal catch-up growth demonstrated significantly less severe outcomes, although their metabolic health was still somewhat impaired when compared to non-growthrestricted controls. ${ }^{57}$ The results of this study demonstrate the detrimental effects of IUGR on the metabolic health of the offspring, as well as the differential effects of accelerated versus normal catch-up growth.

One pathway shown to be involved in the role of pregnancy complications and the offspring's metabolic health later in life is endothelial dysfunction. The endothelium is responsible for the maintenance of proper vascularization of the placenta in pregnancy. ${ }^{58}$ Thus, endothelial dysfunction can be both immediately threatening to the health of the fetus and can have lasting effects on the child's metabolic health. Endothelial cells are responsible for the production of vasoactive substances to maintain vascular homeostasis. ${ }^{37}$ Dysfunction occurs when vascular tissue has a diminished response to nitric oxide-mediated vasodilation, leading to a reduction in blood flow to the fetus. ${ }^{37}$ Significant endothelial dysfunction is thought to be one of the causes of preeclampsia. ${ }^{59}$ Although the pathogenesis of preeclampsia is not well understood, there is agreement that it is at least partially due to inadequate trophoblast invasion of the spiral arteries during placentation. ${ }^{60}$ This is thought to be the result of oxidative stress and inflammation, which trigger endothelial dysfunction in the developing fetus. ${ }^{46}$

Another potential mechanism for the connection between pregnancy complications and offspring metabolic health is that low birth weight and small for gestational age infants often experience a significant period of "catch-up growth" in the postnatal period. These infants have been shown to experience significant changes in metabolism that are associated with an increased risk for obesity. ${ }^{61}$ Given that many pregnancy complications can result in low birth weight or small for gestational age, these conditions represent a high risk population for metabolic syndrome.

\section{Discussion}

Substantial evidence supports the role of the intrauterine environment in determining the metabolic health of the offspring by means of developmental programming. Epidemiologic evidence consistently demonstrates the effects of maternal diet, maternal health, pregnancy complications, and epigenetic modifications on later life metabolic health in the offspring. These phenomena have been further characterized through the use of animal models of developmental programming. The interaction of intrauterine exposures and epigenetic changes results in a complex network of mechanisms that contribute to the development of metabolic syndrome and other metabolic disorders including obesity and T2D.

In this review, we have presented evidence for the relationship between maternal and early postnatal diet, maternal obesity, insulin resistance and metabolic syndrome, pregnancy complications and epigenetic programming and subsequent risk of obesity, diabetes and metabolic syndrome in the offspring. It is clear that many of these factors overlap and may mutually confound these relationships. ${ }^{62}$ For example, maternal and early postnatal diet, along with the maternal metabolic state may confound the relationship between pregnancy complications and subsequent health of the child, which may also be mediated by epigenetic programming. This demonstrates the network of contributors to both developmental programming and the developmental origins of adult disease.

It is also important to consider the potential role of confounders related to social determinants of health. In particular, socioeconomic factors such as access to medical care, income and education level, are related to both prenatal health and pregnancy outcomes. ${ }^{63}$ Such factors present an opportunity to improve the pregnancy environment through public health awareness.

The substantial evidence for the contribution of the intrauterine environment to the future metabolic health of the offspring demonstrates an opportunity for future investigation into the appropriate prenatal diet for optimal maternal and neonatal health. Some studies have shown that adherence to a Mediterranean diet is associated with more favorable 
metabolic health in both the mother and infant, including lower risk for gestational diabetes and low birth weight, as well as lower neonatal insulin, blood glucose, lipids and lipoprotein. ${ }^{64-67}$ These studies demonstrate that a positive intrauterine environment can improve maternal and neonatal outcomes and should be considered for public health recommendations and translation into clinical practice. However, to date, research on modifying maternal diet during pregnancy has primarily been confined to studies using animal models. Many such studies have shown sex differences in the metabolic response to adverse intrauterine conditions, a phenomenon which warrants further investigation. To replicate such findings in humans, observational cohorts should be established to assess the role of the prenatal environment on the future health of the mother and child through adolescence and adulthood.

The growing body of evidence for the relationship between intrauterine environment and metabolic syndrome suggests the need for further investigation of the mechanisms by which prenatal exposures program biological systems to influence future health. Through a better understanding of these mechanisms, we can improve prediction and prevention of pregnancy complications, ultimately improving the health outcomes of mothers and their offspring.

\section{Disclosure}

The authors do not have any conflicts of interest in this work.

\section{References}

1. Tobi EW, Goeman JJ, Monajemi R, et al. DNA methylation signatures link prenatal famine exposure to growth and metabolism. Nat Commun. 2014;5:5592.

2. Hales CN, Barker DJ. Type 2 (non-insulin-dependent) diabetes mellitus: the thrifty phenotype hypothesis. Diabetologia. 1992;35(7):595-601.

3. Alsnes IV, Janszky I, Forman MR, Vatten LJ, Okland I. A population-based study of associations between preeclampsia and later cardiovascular risk factors. Am J Obstet Gynecol. 2014;211(6):657. e1-e7.

4. Barker DJ, Martyn CN, Osmond C, Hales CN, Fall CH. Growth in utero and serum cholesterol concentrations in adult life. BMJ. 1993; 307(6918):1524-1527.

5. Boney CM, Verma A, Tucker R, Vohr BR. Metabolic syndrome in childhood: association with birth weight, maternal obesity, and gestational diabetes mellitus. Pediatrics. 2005;115(3):e290-e296.

6. Leeson CP, Kattenhorn M, Morley R, Lucas A, Deanfield JE. Impact of low birth weight and cardiovascular risk factors on endothelial function in early adult life. Circulation. 2001;103(9):1264-1268.

7. de Boo HA, Harding JE. The developmental origins of adult disease (Barker) hypothesis. Aust N Z J Obstet Gynaecol. 2006;46(1):4-14.

8. Kajantie E, Strang-Karlsson S, Hovi P, et al. Insulin sensitivity and secretory response in adults born preterm: the Helsinki study of very low birth weight adults. J Clin Endocrinol Metab. 2015;100(1):244-250.

9. Koleganova N, Piecha G, Ritz E. Prenatal causes of kidney disease. Blood Purif. 2009;27(1):48-52.

10. Cardozo E, Pavone ME, Hirshfeld-Cytron JE. Metabolic syndrome and oocyte quality. Trends Endocrinol Metab. 2011;22(3):103-109.
11. Robker RL. Evidence that obesity alters the quality of oocytes and embryos. Pathophysiology. 2008;15(2):115-121.

12. Ornoy A. Prenatal origin of obesity and their complications: Gestational diabetes, maternal overweight and the paradoxical effects of fetal growth restriction and macrosomia. Reprod Toxicol. 2011;32(2):205-212.

13. Nistala R, Hayden MR, Demarco VG, Henriksen EJ, Lackland DT, Sowers JR. Prenatal Programming and Epigenetics in the Genesis of the Cardiorenal Syndrome. Cardiorenal Med. 2011;1(4):243-254.

14. Whitelaw NC, Whitelaw E. How lifetimes shape epigenotype within and across generations. Hum Mol Genet. 2006;15 Spec No 2:R131-R137.

15. Hanson MA, Gluckman PD. Early developmental conditioning of later health and disease: physiology or pathophysiology? Physiol Rev. 2014;94(4):1027-1076.

16. Lillycrop KA. Effect of maternal diet on the epigenome: implications for human metabolic disease. Proc Nutr Soc. 2011;70(1):64-72.

17. Bursztyn M, Ariel I. Maternal-fetal deprivation and the cardiometabolic syndrome. J Cardiometab Syndr. 2006;1(2):141-145.

18. Ponzio BF, Carvalho MH, Fortes ZB, do Carmo Franco M. Implications of maternal nutrient restriction in transgenerational programming of hypertension and endothelial dysfunction across F1-F3 offspring. Life Sci. 2012;90(15-16):571-577.

19. Gray C, Vickers MH, Segovia SA, Zhang XD, Reynolds CM. A maternal high fat diet programmes endothelial function and cardiovascular status in adult male offspring independent of body weight, which is reversed by maternal conjugated linoleic acid (CLA) supplementation. PloS One. 2015;10(2):e0115994.

20. Kaar JL, Crume T, Brinton JT, Bischoff KJ, McDuffie R, Dabelea D. Maternal obesity, gestational weight gain, and offspring adiposity: the exploring perinatal outcomes among children study. $J$ Pediatr. 2014;165(3):509-515.

21. Wen LM, Simpson JM, Rissel C, Baur LA. Maternal "junk food” diet during pregnancy as a predictor of high birthweight: findings from the healthy beginnings trial. Birth. 2013;40(1):46-51.

22. Sampey BP, Vanhoose AM, Winfield HM, et al. Cafeteria diet is a robust model of human metabolic syndrome with liver and adipose inflammation: comparison to high-fat diet. Obesity (Silver Spring). 2011;19(6):1109-1117.

23. Fan L, Lindsley SR, Comstock SM, et al. Maternal high-fat diet impacts endothelial function in nonhuman primate offspring. Int J Obes (Lond) 2013;37(2):254-262.

24. Marco A, Kisliouk T, Tabachnik T, Meiri N, Weller A. Overweight and CpG methylation of the Pomc promoter in offspring of high-fat-diet-fed dams are not "reprogrammed" by regular chow diet in rats. FASEB J. 2014;28(9):4148-4157.

25. Briffa JF, McAinch AJ, Romano T, Wlodek ME, Hryciw DH. Leptin in pregnancy and development: a contributor to adulthood disease? Am J Physiol Endocrinol Metab. 2015;308(5):E335-E350.

26. Wild SH, Byrne CD. Evidence for fetal programming of obesity with a focus on putative mechanisms. Nutr Res Rev. 2004;17(2):153-162.

27. Barbero A, Astiz S, Lopez-Bote CJ, et al. Maternal malnutrition and offspring sex determine juvenile obesity and metabolic disorders in a swine model of leptin resistance. PloS One. 2013; 8(10): 78424

28. Shasa DR, Odhiambo JF, Long NM, Tuersunjiang N, Nathanielsz PW, Ford SP. Multigenerational impact of maternal overnutrition/obesity in the sheep on the neonatal leptin surge in granddaughters. Int J Obes (Lond). 2015;39(4):695-701.

29. Bouret SG, Draper SJ, Simerly RB. Trophic action of leptin on hypothalamic neurons that regulate feeding. Science. 2004;304(5667): 108-110.

30. Ferezou-Viala J, Roy AF, Serougne C, et al. Long-term consequences of maternal high-fat feeding on hypothalamic leptin sensitivity and diet-induced obesity in the offspring. Am J Physiol Regul Integr Comp Physiol. 2007;293(3):R1056-R1062.

31. Blue EK, Ballman K, Boyle F, et al. Fetal hyperglycemia and a high-fat diet contribute to aberrant glucose tolerance and hematopoiesis in adult rats. Pediatr Res. 2015;77(2):316-325. 
32. Fisman EZ, Tenenbaum A. Adiponectin: a manifold therapeutic target for metabolic syndrome, diabetes, and coronary disease? Cardiovasc Diabetol. 2014;13:103.

33. Hou M, Chu Z, Liu T, et al. A high-fat maternal diet decreases adiponectin receptor-1 expression in offspring. J Matern Fetal Neonatal Med. 2015;28(2):216-221.

34. Doblado M, Moley KH. Glucose metabolism in pregnancy and embryogenesis. Curr Opin Endocrinol Diabetes Obes. 2007;14(6): 488-493.

35. Thakali KM, Saben J, Faske JB, et al. Maternal pregravid obesity changes gene expression profiles toward greater inflammation and reduced insulin sensitivity in umbilical cord. Pediatr Res. 2014;76(2):202-210.

36. West NA, Crume TL, Maligie MA, Dabelea D. Cardiovascular risk factors in children exposed to maternal diabetes in utero. Diabetologia. 2011;54(3):504-507.

37. Fernandez-Twinn DS, Ozanne SE. Mechanisms by which poor early growth programs type-2 diabetes, obesity and the metabolic syndrome. Physiol Behav. 2006;88(3):234-243.

38. Yogev Y, Visser GH. Obesity, gestational diabetes and pregnancy outcome. Semin Fetal Neonatal Med. 2009;14(2):77-84.

39. Yessoufou A, Moutairou K. Maternal diabetes in pregnancy: early and long-term outcomes on the offspring and the concept of "metabolic memory". Exp Diabetes Res. 2011;2011:218598.

40. O'Brien T, Nguyen TT, Zimmerman BR. Hyperlipidemia and diabetes mellitus. Mayo Clin Proc. 1998;73(10):969-976.

41. Li CC, Young PE, Maloney CA, et al. Maternal obesity and diabetes induces latent metabolic defects and widespread epigenetic changes in isogenic mice. Epigenetics. 2013;13;8(6):602-611.

42. Xu J, Ye J, Wu Y, et al. Reduced fetal telomere length in gestational diabetes. PloS One. 2014;9(1):e86161.

43. Dahlhoff M, Pfister S, Blutke A, et al. Peri-conceptional obesogenic exposure induces sex-specific programming of disease susceptibilities in adult mouse offspring. Biochim Biophys Acta. 2014;1842(2):304-317.

44. Hwang LL, Wang CH, Li TL, et al. Sex differences in high-fat dietinduced obesity, metabolic alterations and learning, and synaptic plasticity deficits in mice. Obesity (Silver Spring). 2010;18(3):463-469.

45. Fernandez-Twinn DS, Alfaradhi MZ, Martin-Gronert MS, et al. Downregulation of IRS-1 in adipose tissue of offspring of obese mice is programmed cell-autonomously through post-transcriptional mechanisms. Mol Metab. 2014;3(3):325-333.

46. Leduc L, Levy E, Bouity-Voubou M, Delvin E. Fetal programming of atherosclerosis: possible role of the mitochondria. Eur JObstet Gynecol Reprod Biol. 2010;149(2):127-130.

47. Tenhola S, Rahiala E, Halonen P, Vanninen E, Voutilainen R. Maternal preeclampsia predicts elevated blood pressure in 12-year-old children: evaluation by ambulatory blood pressure monitoring. Pediatr Res. 2006;59(2):320-324.

48. Miettola S, Hartikainen AL, Vaarasmaki M, et al. Offspring's blood pressure and metabolic phenotype after exposure to gestational hypertension in utero. Eur J Epidemiol. 2013;28(1):87-98.

49. Lawlor DA, Macdonald-Wallis C, Fraser A, et al. Cardiovascular biomarkers and vascular function during childhood in the offspring of mothers with hypertensive disorders of pregnancy: findings from the Avon Longitudinal Study of Parents and Children. Eur Heart J. 2012; 33(3):335-345.
50. Kvehaugen AS, Dechend R, Ramstad HB, Troisi R, Fugelseth D, Staff AC. Endothelial function and circulating biomarkers are disturbed in women and children after preeclampsia. Hypertension. 2011;58(1): 63-69.

51. He J, Zhang A, Fang M, et al. Methylation levels at IGF2 and GNAS DMRs in infants born to preeclamptic pregnancies. BMC Genomics. $2013 ; 14: 472$.

52. Cianfarani S, Germani D, Branca F. Low birthweight and adult insulin resistance: the "catch-up growth" hypothesis. Arch Dis Child Fetal Neonatal Ed. 1999;81(1):F71-F73.

53. Hermann GM, Dallas LM, Haskell SE, Roghair RD. Neonatal macrosomia is an independent risk factor for adult metabolic syndrome. Neonatology. 2010;98(3):238-244.

54. Seki Y, Williams L, Vuguin PM, Charron MJ. Minireview: Epigenetic programming of diabetes and obesity: animal models. Endocrinology. 2012;153(3):1031-1038.

55. Ozanne SE. Metabolic programming in animals. Br Med Bull. 2001;60: 143-152.

56. Jansson T, Lambert GW. Effect of intrauterine growth restriction on blood pressure, glucose tolerance and sympathetic nervous system activity in the rat at 3-4 months of age. J Hypertens. 1999;17(9):1239-1248.

57. Dellschaft NS, Alexandre-Gouabau MC, Gardner DS, et al. Effect of pre- and postnatal growth and post-weaning activity on glucose metabolism in the offspring. $J$ Endocrinol. 2015;224(2):171-182.

58. Slavik L, Prochazkova J, Prochazka M, Simetka O, Hlusi A, Ulehlova J. The pathophysiology of endothelial function in pregnancy and the usefulness of endothelial markers. Biomed Pap Med Fac Univ Palacky Olomouc Czech Repub. 2011;155(4):333-337.

59. Bellamy L, Casas JP, Hingorani AD, Williams DJ. Pre-eclampsia and risk of cardiovascular disease and cancer in later life: systematic review and meta-analysis. BMJ. 2007;335(7627):974.

60. Redman CW, Sargent IL. Latest advances in understanding preeclampsia. Science. 2005;308(5728):1592-1594.

61. Stocker CJ, Cawthorne MA. The influence of leptin on early life programming of obesity. Trends Biotechnol. 2008;26(10):545-551.

62. Mamun AA. Maternal obesity during pregnancy is associated with adult offspring cardiovascular morbidity and mortality but may represent confounding by other factors. Evid Based Med. 2014;19(3):111.

63. Snelgrove JW, Murphy KE. Preterm birth and social inequality: Assessing the effects of material and psychosocial disadvantage in a UK birth cohort. Acta Obstet Gynecol Scand. Epub April 4, 2015.

64. Tobias DK, Zhang C, Chavarro J, et al. Prepregnancy adherence to dietary patterns and lower risk of gestational diabetes mellitus. Am J Clin Nutr. 2012;96(2):289-295.

65. Timmermans S, Steegers-Theunissen RP, Vujkovic M, et al. The Mediterranean diet and fetal size parameters: the Generation R Study. $\mathrm{Br} J$ Nutr. 2012;108(8):1399-1409.

66. Gesteiro E, Rodriguez Bernal B, Bastida S, Sanchez-Muniz FJ. Maternal diets with low healthy eating index or Mediterranean diet adherence scores are associated with high cord-blood insulin levels and insulin resistance markers at birth. Eur J Clin Nutr. 2012;66(9):1008-1015.

67. Gesteiro E, Bastida S, Rodriguez Bernal B, Sanchez-Muniz FJ. Adherence to Mediterranean diet during pregnancy and serum lipid, lipoprotein and homocysteine concentrations at birth. Eur J Nutr. Epub November 20, 2014.

Diabetes, Metabolic Syndrome and Obesity: Targets and Therapy

\section{Publish your work in this journal}

Diabetes, Metabolic Syndrome and Obesity: Targets and Therapy is an international, peer-reviewed open-access journal committed to the rapid publication of the latest laboratory and clinical findings in the fields of diabetes, metabolic syndrome and obesity research. Original research, review, case reports, hypothesis formation, expert opinion and commentaries are all considered for publication. The manuscript management system is completely online and includes a very quick and fair peer-review system, which is all easy to use. Visit http://www.dovepress.com/testimonials.php to read real quotes from published authors. 